\title{
A murine macrofilaricide pre-clinical screening model for onchocerciasis and lymphatic filariasis
}

\author{
Alice Halliday ${ }^{1}$, Ana F Guimaraes ${ }^{1}$, Hayley E Tyrer ${ }^{1}$, Haelly Mejane Metuge ${ }^{2}$, Chounna Ndongmo Winston Patrick ${ }^{2,3}$, \\ Kengne-Ouafo Jonas Arnaud ${ }^{2,3}$, Tayong Dizzle Bita Kwenti ${ }^{2,3}$, George Forsbrook', Andrew Steven', Darren Cook', \\ Peter Enyong ${ }^{2}$, Samuel Wanji ${ }^{2,3}$, Mark J Taylor ${ }^{1}$ and Joseph D Turner ${ }^{1 *}$
}

\begin{abstract}
Background: New drugs effective against adult filariae (macrofilaricides) would accelerate the elimination of lymphatic filariasis and onchocerciasis. Anti-Onchocerca drug development is hampered by the lack of a facile model. We postulated that SCID mice could be developed as a fmacrofilaricide screening model.

Methods: The filaricides: albendazole (ABZ), diethylcarbamazine (DEC), flubendazole (FBZ), ivermectin (IVM) and the anti-Wolbachia macrofilaricide, minocycline (MIN) were tested in Brugia malayi (Bm)-parasitized BALB/c SCID mice vs vehicle control (VC). Responses were compared to BALB/c wild type (WT). Onchocerca ochengi male worms or onchocercomata were surgically implanted into BALB/C SCID, CB.17 SCID, BALB/C WT mice or Meriones gerbils. Survival was evaluated at 7-15 days. BALB/C SCID were tested to evaluate the responsiveness of pre-clinical macrofilaricides FBZ and rifapentine (RIFAP) against male Onchocerca.
\end{abstract}

Results: $W T$ and SCID responded with $>95 \%$ efficacy following ABZ or DEC treatments against $B m$ larvae $(P<0.0001)$. IVM was partially filaricidal against Bm larvae in WT and SCID (WT; 39.8\%, $P=0.0356$ and SCID; $56.7 \%, P=0.026$ ). SCID responded similarly to WT following IVM treatment of microfilaraemias (WT; 79\%, $P=0.0194$. SCID; 76\%, $P=0.0473$ ). FBZ induced a total macrofilaricidal response against adult $B m$ in WT and SCID (WT; $P=0.0067, \mathrm{SCID} ; P=0.0071)$. MIN induced a $>90 \%$ reduction in $B m$ Wolbachia burdens $(P<0.0001)$ and a blockade of microfilarial release $(P=0.0215)$ in SCID. Male Onchocerca survival was significantly higher in SCID vs WT mice, but not gerbils, after +15 days (60\% vs $22 \%$ vs $39 \% P=0.0475)$. Onchocercoma implants had engrafted into host tissues, with evidence of neovascularisation, after +7 days and yielded viable macro/microfilariae ex vivo. FBZ induced a macrofilaricidal effect in Onchocerca male implanted SCID at +5 weeks (FBZ; $1.67 \%$ vs VC; $43.81 \%, P=0.0089$ ). Wolbachia loads within male Onchocerca were reduced by $99 \%$ in implanted SCID receiving RIFAP for +2 weeks.

Conclusions: We have developed a 'pan-filarial' small animal research model that is sufficiently robust, with adequate capacity and throughput, to screen existing and future pre-clinical candidate macrofilaricides. Pilot data suggests a murine onchocercoma xenograft model is achievable.

Keywords: Anti-filarial, Lymphatic filariasis, Onchocerciasis, Macrofilaricide, Brugia, Onchocerca, Wolbachia

\section{Background}

The tissue dwelling filaria, Onchocerca volvulus, infects an estimated 37 million people, predominantly in SubSaharan Africa [1]. Ocular onchocerciasis (river blindness) is the second leading cause of worldwide preventable blindness [2] and is a priority neglected tropical disease (NTD) targeted for elimination [3]. Current treatments

\footnotetext{
* Correspondence: joseph.turner@lstmed.ac.uk

${ }^{1}$ Department of Parasitology, Liverpool School of Tropical Medicine, Liverpool, UK Full list of author information is available at the end of the article
}

with ivermectin (IVM) target the transmissive stages of O. volvulus, microfilariae ( $\mathrm{mf}$ ), in the skin, with limited efficacy against adult (macrofilariae) fertility or survival [4]. Whilst effective at reducing disease, due to repopulation of the skin with mf post-treatment, IVM has to be administered periodically. In addition, IVM has to be delivered for protracted periods (17-20+ years) due to the longevity of macrofilarial infections (10-15 years). This presents a challenge in sustainability with high population coverage in mass drug administration programmes (MDA) to 
maintain control of disease and eventually break the transmission cycle. Further, the emergence of sub-optimal responders to IVM following repetitive treatments [5] and the risk of severe adverse reactions in patients co-infected with the bloodborne filaria, Loa loa [6], exert additional challenges on maintaining control of onchocerciasis.

Drugs effective at killing adult Onchocerca (macrofilaricides), would be desirable to address this pressing global health problem and accelerate the ultimate elimination of onchocerciasis. New macrofilaricides may well also have useful indications against the causative agents of lymphatic filariasis; LF (Wuchereria bancrofti, Brugia malayi, B. timori), especially to reduce the long tail of 'endgame mop-up' in countries that have completed extensive elimination programmes or in 'hard to reach' areas [1].

Existing Onchocerca macrofilaricides have either too low selective toxicity (suramin) [7], are known to induce severe adverse reactions contraindicating usage at doses required to be effective (e.g. diethylcarbamazine or high dose ivermectin) $[8,9]$ or presently cannot be delivered orally (e.g. flubendazole) [10]. Whilst targeting the O. volvulus endosymbiont, Wolbachia, with tetracycline antibiotics has proven safe in delivering macrofilaricidal activity, including in individuals co-infected with $L$. loa [11,12], present protracted treatment lengths (4-6 weeks) and contraindications in certain population groups (pregnant women and children $<8$ years old) precludes the wide-scale use of this class of anti-Wolbachia drug in large scale MDA [1].

Stimulated by renewed philanthropic investment by a number of stakeholders, a number of drug discovery and development programmes have been initiated to identify new candidate macrofilaricidal therapeutics that can effectively and safely target the macrofilarial stage of $O$. volvulus or its endosymbiont in a timeframe compatible with MDA delivery (generally considered an orally administered course not exceeding seven days).

The development of Onchocerca macrofilaricides is hindered by the lack of an appropriate small animal laboratory model to robustly evaluate candidates in vivo at the pre-clinical stage. A long term model of brugian lymphatic filariasis (B. malayi/B. pahangi) is available in the susceptible gerbil host Meriones unguiculatus [13] and certain strains of inbred mice are susceptible to the filaria, Litomosoides sigmodontis [14]. Whilst these rodent filariasis models are undoubtedly useful in identifying efficacious filaricidal compounds, overt differences in the biology of Onchocerca species requires confirmation of effectiveness at the pre-clinical stage before informed decisions can be made about clinical development for onchocerciasis indications. A current example of this has been the lack of translation of macrofilaricidal activity of the macrocyclic lactone, moxidectin, from pre-clinical testing against Brugia in gerbils and dogs, when evaluated by a phase II clinical trial in onchocerciasis patients $[15,16]$. Traditionally, the use of cattle naturally infected with bovine Onchocerca (O. dukei, O. gutterosa, O. ochengi, O. lienalis) has been exploited as a pre-clinical system to scrutinise Onchocerca-specific macrofilaricidal activity. Throughput is severely restricted by the logistics of identifying and experimenting on parasitized cattle in subSaharan Africa and the large quantities of drug required to dose up to 0.5 metric tonne animals. Further, without challenging empirical pharmacological evaluation, requiring robust sample sizes, atypical pharmacokinetic profiles within ruminants may lead to erroneous conclusions regarding efficacy.

A potential solution to the current challenges facing pre-clinical onchocerciasis macrofilaricide evaluation is the development of a small animal model of macrofilarial onchocerciasis. Presently, only larval Onchocerca life cycle stages are routinely used to screen filaricidal drug candidates in mice. Infectious stage (L3) larvae of O. volvulus can be implanted within micro-chambers to achieve only abbreviated development to the L4 larval stage [17]. Microfilarial infections of the skin, utilising purified cattle Onchocerca mf, can be established in inbred laboratory mouse strains and have been utilised in pre-clinical filaricidal testing [18]. However, no reliable macrofilarial Onchocerca small animal model has been described. Previous attempts to utilize inbred mouse strains, including lymphopenic strains, to establish Onchocerca macrofilarial infections from infectious stage (L3) larval inoculations, has thus far proven unsuccessful [19]. However, Rajan and colleagues demonstrated that $O$. volvulus female worm 'loops', exposed from surrounding nodular encasement in excised nodules, could remain viable following implantation into Severe-Combined ImmunoDeficiency (SCID) mice [20]. The same laboratory has also demonstrated that full development of Brugia can occur in SCID strains [21]. Further, our laboratory has previously demonstrated that $O$. lienalis $\mathrm{mf}$ infections can persist for at least 100 days in SCID recipients without waning [22].

Thus, in this paper, we evaluated the suitability of the SCID mouse as a pre-clinical model to test macroflaricidal activity against filariae, using laboratory maintained Brugia malayi to assess responsiveness to a range of filaricidal compounds before trialling survival and drug responses against Onchocerca ochengi implants.

\section{Methods}

\section{Animals}

Male BALB/c SCID were purchased from Harlan Laboratories, UK, while male CB.17 SCID mice and BALB/C WT mice were purchased from Charles River, UK. Male Meriones unguiculatus (Mongolian gerbils; jirds) were purchased from either Charles River, UK or Janvier Laboratories, France. Rodents shipped to REFOTDE, Buea, Cameroon, were maintained in conventional housing with 
daily cage cleaning and changing of food. Food, water and bedding were sterilised by autoclaving. For B. malayi experiments, animals were kept at the Biomedical Services Unit (BSU), University of Liverpool, UK in specific pathogen-free (SPF) conditions. All experiments carried out in Cameroon were approved by the Animal Care Committee, REFOTDE. All experiments on animals in the UK were approved by the ethical committees of the University of Liverpool and LSTM, and were conducted according to Home Office (UK) requirements.

\section{Brugia malayi parasites}

The life cycle of $B$. malayi $(B m)$ was maintained in mosquitoes and susceptible Meriones gerbils at LSTM. To generate infective $B m$ larvae ( $B m \mathrm{~L} 3$ ) for infections, female adult Aedes aegypti mosquitoes were fed with $\mathrm{Bm}$ microfilariae $(\mathrm{mf})$ collected from infected gerbils by catheterisation, as previously described [23], followed by mixing with human blood and feeding through an artificial membrane feeder $\left(\right.$ Hemotek $\left.^{\odot}\right)$. Blood-fed mosquitoes were reared for 14 days to allow for development to L3. The L3 were collected from infected mosquitoes by crushing and concentration using a Baermann's apparatus and RPMI medium.

\section{Onchocerca ochengi parasites}

$O$. ochengi nodules were obtained from the skins of naturally infected zebu cattle from the Adamawa region of Cameroon through existing commercial practice for meat production. At abbatoirs in the South West Province, Cameroon, female cattle were checked for $O$. ochengi infection by palpation of the skin of the umbilical region for onchocercomata. Positive skins were collected after slaughter. The tissue was then transported to REFOTDE, Buea within 2 hours of collection. Skins were washed several times and the hair was removed by shaving. Onchocercomata were excised from dermal tissue using sterile scalpels and forceps, and were placed in RPMI containing penicillin, streptomycin and neomycin (RPMI + PSN). To obtain free adult males, onchocercomata were cut gently to expose the adult worms using sterile scalpels and were incubated in petri dishes containing RPMI + PSN for 4 hours at $37^{\circ} \mathrm{C}$, $5 \% \mathrm{CO}_{2}$, to allow males to escape into the medium. Free, intact and motile adult males were confirmed by visualisation of posterior anatomy, were washed several times in fresh RPMI + PSN and kept at $37^{\circ} \mathrm{C}, 5 \% \mathrm{CO}_{2}$ overnight.

\section{B. malayi experimental infections}

For B. malayi $(\mathrm{Bm})$ L3 infection, 100 freshly collected, motile $B m$ L3 were injected via the intra-peritoneal route. Efficiencies of inoculations were confirmed by needle washout. For $\mathrm{mf}$ perfusion, $\mathrm{Bm} \mathrm{mf}$ were harvested by peritoneal washings of patently infected Meriones gerbils under isoflurane anaesthesia, washed in RMPI + PSN and purified by PD10 column size exclusion chromatography (Amersham). Bm $\mathrm{mf}$ were enumerated by microscopy, concentrated by centrifugation and $0.125 \times 10^{6} \mathrm{mf}$ were inoculated into the circulation via the lateral tail vein as described previously [24].

\section{Implantation of adult macrofilariae}

$\mathrm{Bm}$ macrofilariae were collected from infected SCID mice and were grouped into batches of 13 adults. Male BALB/c SCID and BALB/C WT mice were placed under surgical anaesthesia using isofluorane and were given s.c. injection of bruprenorphine before $13 \mathrm{Bm}$ macrofilariae were placed into the peritoneal cavity by making a small incision to the skin and abdominal cavity wall in the upper right quadrant. The incisions were re-sutured after implant and animals were individually housed after surgery.

For Onchocerca implants, rodents were placed under surgical anaesthesia using i.p. injections of ketamine and medetomidine. Onchocercomata were thoroughly cleaned of bovine tissue, washed in several changes of RPMI + PSN and small sections of the adult worms were exposed by partial rupture of the capsule. Groups of 8-15 motile $O$. ochengi male macrofilariae or 4 prepared $O$. ochengi nodules were implanted into the peritoneal cavity or to the cutaneous tissue of the upper side of the neck (for s.C nodule implants). All wounds were re-sutured after surgery and animals were individually housed and closely monitored for the recovery period (7 days post-op).

\section{Drug treatments}

The drug doses in $\mathrm{mg} / \mathrm{kg}$ and routes used in this study were: Albendazole (ABZ; $50 \mathrm{mg} / \mathrm{kg}$ qd po), diethylcarbamazine (DEC; $50 \mathrm{mg} / \mathrm{kg}$ qd po), flubendazole (FBZ; $10 \mathrm{mg} /$ $\mathrm{kg}$ qd sc), ivermectin (IVM; $5 \mathrm{mg} / \mathrm{kg}$ qd ip or $15 \mathrm{mg} / \mathrm{kg} \mathrm{qd}$ po), minocycline (MIN; $25 \mathrm{mg} / \mathrm{kg}$ bid po) and rifapentine (RIFAP; $15 \mathrm{mg} / \mathrm{kg}$ qd po). Drugs were dissolved in standard suspension vehicle $(0.5 \%$ sodium carboxymethyl cellulose; $0.5 \%$ benzyl alcohol; $0.4 \%$ Tween $80 ; 0.9 \% \mathrm{NaCl}$ ), with the exception of MIN, which was dissolved in water, RIFAP which was dissolved in 55\% polyethylene glycol 300; $25 \%$ propylene glycol; $20 \%$ water and parenteral IVM which was dissolved in $1 \%$ DMSO. Oral drugs were administered in volumes of $100-200 \mu$ l by gavage. All drugs were purchased from Sigma Aldrich.

\section{Drug efficacy assessments}

Developing larvae, macrofilariae and released $\mathrm{mf}$ were recovered by peritoneal washings and enumerated by microscopy. Motile, unencapsulated worms were scored as viable. $B m \mathrm{mf}$ were centrifuged before being resuspended in a known volume and a sample enumerated. For $B m \mathrm{mf}$ microfilaraemias, $30 \mu \mathrm{l}$ thick films on uncoated glass slides (Corning) were prepared from freshly-collected blood (tail bleeds or cardiac punctures post-mortem) in $5000 \mathrm{U} / \mathrm{ml}$ 
lithium heparin anti-coagulant (Sigma Aldrich). Slides were air dried, de-haemaglobinised in tap water, fixed in $80 \% \mathrm{MeOH}$ and stained with Giemsa (BDH). $\mathrm{Bm}$ mf were enumerated by microscopy. For recovery of $O$. ochengi nodules, the nodule tissue was excised from the area of tissue engraftment post-mortem. Nodules were dissected and incubated in RPMI $+\mathrm{PSN}, 37^{\circ} \mathrm{C}, 5 \% \mathrm{CO}_{2}$ for $+4 \mathrm{~h}$ before the presence of motile worms or $\mathrm{mf}$ was evaluated by microscopy.

\section{PCR quantification of Wolbachia}

Individual adult filariae were fixed in RNAlater and stored at $4{ }^{\circ} \mathrm{C}$. For Wolbachia enumerations, DNA was extracted from worm samples using the DNeasy Blood and Tissue Kit (Qiagen) according to manufacturer's instructions. Levels of Bm Wolbachia wsp and Bm gst gene copy numbers were quantified using qPCR with $B m$-specific primers, as reported elsewhere [25]. Levels of $O$. ochengi-specific wsp and gst were estimated using identical PCR conditions, with the $w s p$ primer pairs: wsp420TGTTGGT(AG)TTGGT(GC)TTGGTG, wsp583 AACCAAA(AG)TAGCGAGC(CT)CCA and the gst primer pairs: gst175ATTGAAGCGCTTATTAGTCTGC, gst3 05TGTCGTTTCCATTTCATTTTC.

\section{Statistical analysis}

Where intra-group data was skewed, non-parametric analyses were used to compare statistical differences between two independent groups (Mann Whitney test) or three or more independent groups (Kruskal Wallis with Dunn's post-hoc tests). Where intra-group data displayed a normal distribution, parametric 1way ANOVA with Bonferroni post-hoc tests were used to examine statistical differences between 3 or more groups. Paired $\mathrm{T}$ tests evaluated changes in time within individual animals. Correlations were assessed for significance using Pearson Correlation test. Significance is indicated by $\mathrm{P}<$ $0.05^{*}, \mathrm{P}<0.01^{* *}$ and $\mathrm{P}<0.001^{* * * *}$.

\section{Results}

\section{Evaluating the performance of reference filaricidal} compounds against $B$. malayi in SCID mice

We adapted mouse models of Brugian filariasis [26-28] to scrutinise whether the standard anti-filarial drugs (SAFD) albendazole (ABZ), diethylcarbamazine (DEC) and ivermectin (IVM) or drugs with demonstrated macrofilaricidal properties, flubendazole (FBZ) and the tetracycline antiwolbachia antibiotic, minocycline (MIN), displayed predictable efficacy in a Severe-Combined Immuno-Deficiency (SCID) mouse model system.

For SAFD, which predominantly target the larval stages of filariae in clinical and veterinary indications, we inoculated either $\mathrm{BALB} / \mathrm{c}$ SCID or $\mathrm{BALB} / \mathrm{c}$ wild type (WT) mice, as an immune-sufficient control, with 100 infectious third stage (L3) of Brugia malayi $(\mathrm{Bm})$ via the intra-peritoneal route. Infected mice were orally administered with SAFD or vehicle control (VC) at indicated doses for seven days, before $\mathrm{Bm}$ larvae were recovered by peritoneal lavage $(+24 \mathrm{~h}$ following the final drug exposure) and motile parasites enumerated. Results are shown in Figure 1. ABZ and DEC treatment delivered an almost complete filaricidal activity in WT mice (100\% and $>99 \%$, respectively). These potent filaricidal efficacies were emulated within SCID hosts (ABZ; 98\%, DEC; $100 \%$ reductions, $P<0.0001)$. IVM was partially filarici$\mathrm{dal}$ at the dose administered $(15 \mathrm{mk} / \mathrm{kg} \mathrm{qd})$ in WT mice (39.8\% mean reduction, $P=0.0356$ ). In SCID mice, a partial filaricidal response was also evident (56.7\% mean reduction, $P=0.026$ ).

To further assess responses of the SAFD, IVM, against $\mathrm{mf}$ in the circulation, $\mathrm{Bm} \mathrm{mf}$ were inoculated via the tail vein into $B A L B / c$ SCID mice or $B A L B / c$ immune competent controls. Initial parasitaemias were measured at a baseline of $+48 \mathrm{~h}$ after inoculation, before groups of mice were administered with a single parenteral dose of 5MK IVM or VC. IVM induced rapid reductions in microfilaraemia $+24 \mathrm{~h}$ post-treatment and levels further declined $+7 \mathrm{~d}$ post-treatment in both WT and SCID mice (79\%; $P=0.0194$ and $76 \% ; P=$ 0.0473 median reductions $+7 \mathrm{~d}$, respectively, Figure $2 \mathrm{~A}$ ). In SCID mice, the levels of $\mathrm{mf}$ recovered from the cardiac puncture at termination were also significantly reduced in IVM-treated mice (89\% median reduction, $P=0.0286$, Figure 2B).

Whilst infectious stage $B m$ L3 are reported to develop into mf-producing macrofilariae in SCID mice, WT mice are refractory to full $\mathrm{Bm}$ development $[21,26]$. For the purposes of validating direct FBZ macrofilaricidal efficacy in immunodeficient mice compared with immunosufficient controls, we implanted a defined number of $\mathrm{Bm}$ macrofilariae sourced from +35 day ip infections of SCID donors into either WT or SCID recipients. Following +2 day recovery from surgery, mice were placed on parenteral FBZ suspension $(10 \mathrm{mk} / \mathrm{kg}$ subcutaneous, qd) or matching VC for a total of five days and macrofilaricidal efficacy was evaluated +5 weeks following the last drug exposure. Comparing VC groups, there was a marginally non-significant decreased recovery of $\mathrm{Bm}$ macrofilariae in $\mathrm{BALB} / \mathrm{c} \mathrm{WT}$ versus $\mathrm{BALB} / \mathrm{c}$ SCID mice $(P=0.069)$. FBZ treatments were $100 \%$ efficacious in both WT and SCID BALB/c mice implanted with $\mathrm{Bm}$ macrofilariae (Figure 3A; average recovery of $0 \% \mathrm{FBZ}$ treatment vs. $38 \%, P=0.0067$ for VC WT and $46 \% P=0.0071$ for VC SCID). Entrapped immotile macrofilariae encapsulated within leukocytic granulomas were frequently evident in WT but not SCID mice (median recovery 4, WT vs 0, SCID, $P=0.0104$; Figure $3 \mathrm{~B})$. 


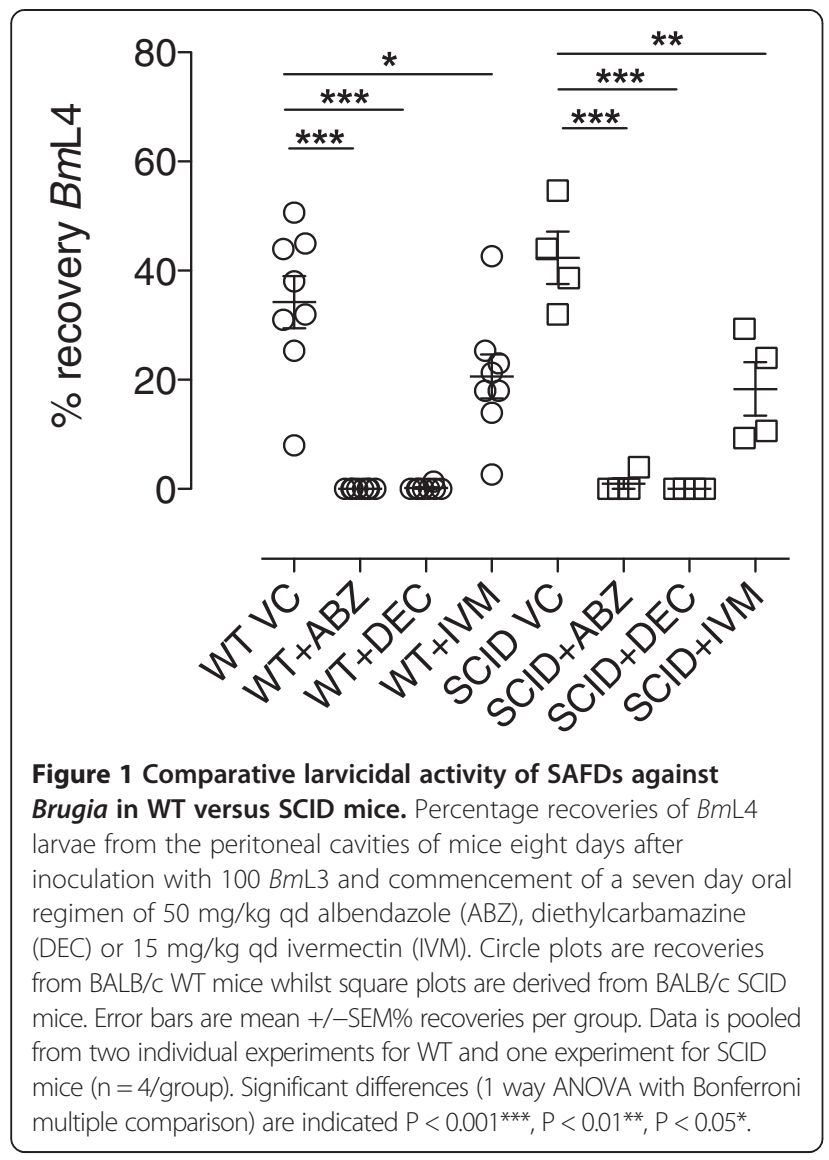

We took advantage of the full development of $\mathrm{Bm}$ in SCID mice to examine the response to oral treatment with the anti-Wolbachia tetracycline, MIN, against the macrofilarial stage of the parasite. SCID BALB/c mice were infected with 100 infectious stage $B m L 3$ larvae ip and dosing with MIN or vehicle control (VC) commenced at $+6 \mathrm{wk}$ post-infection, when parasites had reached the juvenile adult stage. Mice were left for two weeks following the last drug exposure and adult $\mathrm{Bm}$ and released $\mathrm{mf}$ were collected at the early onset of patency $(+12 \mathrm{wks}$ post-infection). The average recovery of adult $\mathrm{Bm}$ in control mice was 9.4 +/-2.6 SEM $(\mathrm{n}=5)$ and recoveries did not vary in MIN treated animals (Figure 4A). Numbers of Wolbachia were quantified following DNA extraction of individual female worms ( $n=10 /$ group, pooled from individual mice; Figure 4B). In the control group, average Wolbachia numbers per female were $5.865 \times 10^{7}+/-0.72 \times 10^{7}$. The average number of Wolbachia in female worms exposed to MIN in vivo in SCID mice had been significantly reduced to $1.837 \times$ $10^{6}+/-0.702 \times 10^{6}$. This equated to a $98.4 \%$ reduction in Wolbachia $(P<0.0001)$. The majority of control mice also contained peritoneal $\mathrm{mf}$ (4/5 mice; Figure $4 \mathrm{C})$. Mf production was enumerated per female worm and, on average, each 12 week old female worm had produced
$373.3(+/-204.4) \mathrm{mf}$. Contrastingly, there was a complete absence of $\mathrm{mf}$ in the peritoneal cavity of MIN treated SCID mice $(P=0.0215)$, consistent with loss of embryonic development following the sterilisation of Wolbachia from adult $\mathrm{Bm}$ filarial tissues.

\section{Yields of Onchocerca macrofilariae from naturally parasitized cattle}

Zebu cattle, naturally infected with the bovine Onchocerca species, $O$. ochengi, were identified within herds moved from pasture land in the Adamawa Region and slaughtered for meat production in abattoirs located in the South West region of Cameroon. Over a 48-day collection period, a total of 2612 nodules were collected from 28 infected hides (mean 105.5 +/- 28.4 SEM, Figure 5), which were processed to purify motile male Onchocerca macrofilariae. A positive correlation between numbers of nodules recovered and numbers of adult males was observed (Pearson $\mathrm{R}=0.869 ; P<0.0001$ ) and, on average, $69.7+$ - 16.4 SEM motile male macrofilariae were recovered per infected cattle hide processed. This was equivalent to an average ratio of $0.66: 1$ male macrofilariae recovered per harvested nodule.

\section{Onchocerca macrofilariae survival following implantation into WT/SCID mice and Mongolian gerbils}

Using the intraperitoneal surgical implant technique as detailed above for $\mathrm{Bm}$, we implanted isolated motile male Onchocerca macrofilariae into various laboratory rodent models and evaluated survival following +15 days (Figure 6). As well as comparison of WT vs SCID mice, we tested survival in Mongolian gerbils (Meriones unguiculatus), an outbred laboratory rodent susceptible to a number of other filariae. After 15 days post-surgical implant, we successfully recovered parasites from all rodent recipients with the exception of one WT BALB/c mouse. Male Onchocerca macrofilariae recovered from rodent implants were monitored for viability ex vivo in culture after recovery from rodent hosts and motility comparable to freshly isolated adult males was observable up to 7 days. At +15 days, recoveries of male macrofilariae were, on average, significantly lower in WT BALB/c versus SCID BALB/c mice (22\% vs $60 \%$ median recovery, $P=0.0475$ ), whilst the average recovery of Onchocerca male macrofilariae was not significantly different in Meriones gerbils vs WT or SCID BALB/c mice (38\%).

We also trialled the implantation of isolated $O$. ochengi onchocercomata, containing a mixture of cow tissue plus both female and male Onchocerca macrofilariae, into SCID $\mathrm{BALB} / \mathrm{c}$ or SCID CB.17 strains. In this pilot study, four onchocercomata were implanted into the peritoneal cavity or sub-cutaneously at the nape of the neck of each SCID recipient. Table 1 and Figure 7 detail the parasitological observations +7 days 


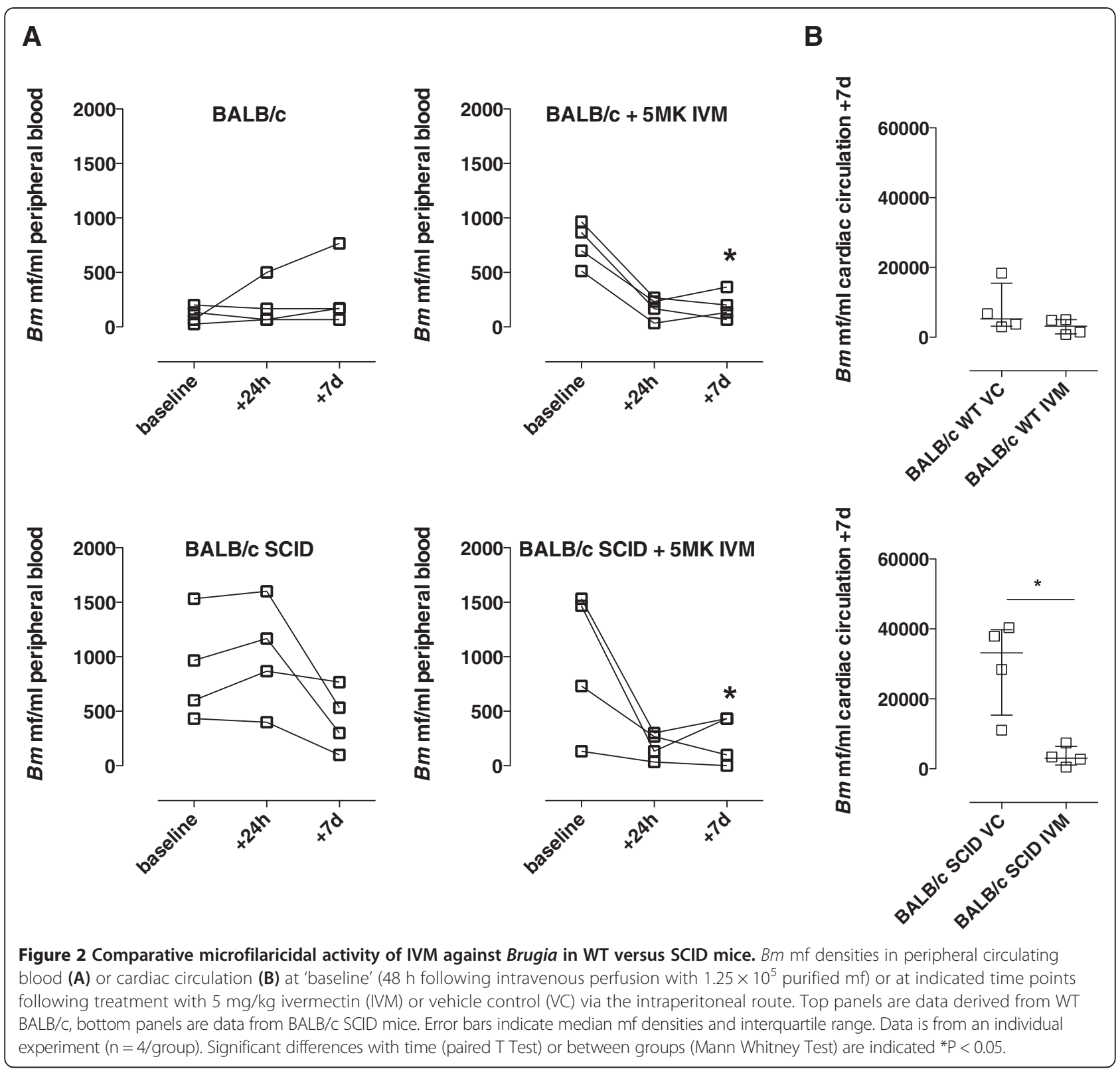

following nodule implantation. At necropsy, the majority of SCID BALB/c recipients (4/6) had evidence of engraftment of some or all implanted onchocercomata into abdominal visceral tissues, mainly connective tissues continuous with the mesenteries and visceral fat pads. In engrafted onchocercomata, neovacularisation could be observed (9/24 implanted nodules), manifest as capillary beds distributed across the capsule surface, stemming from proximal host vascular networks. Onchocercomata were extracted, partially dissected to expose loops of female macrofilariae and cultured ex vivo for 4 hours. Observations of motility during this period revealed that in 5/ 6 SCID recipients, $100 \%$ of implanted nodules contained motile female Onchocerca macrofilariae. Transplanted onchocercomata yielded motile male Onchocerca macrofilariae after recovery from 4/6 SCID recipients at a ratio similar to that observed from freshly isolated nodules (i.e. 1 male/2 nodules). Following sub cutaneous implantations in SCID CB.17 mice, $100 \%$ of onchocercomata $(n=8)$ in $2 / 2$ recipients displayed engraftment into the sub cutaneous layer with evidence of neovascularisation. Vessel-like structures containing blood cells, proximal to female worms, were identifiable within implanted onchocercomata by histology. All onchocercomata recovered from sub cutaneous implants at +7 days contained motile female macrofilariae when dissected and cultured ex vivo. A single motile male macrofilaria was released from nodules derived from $1 / 2$ recipient mice. Evidence of 


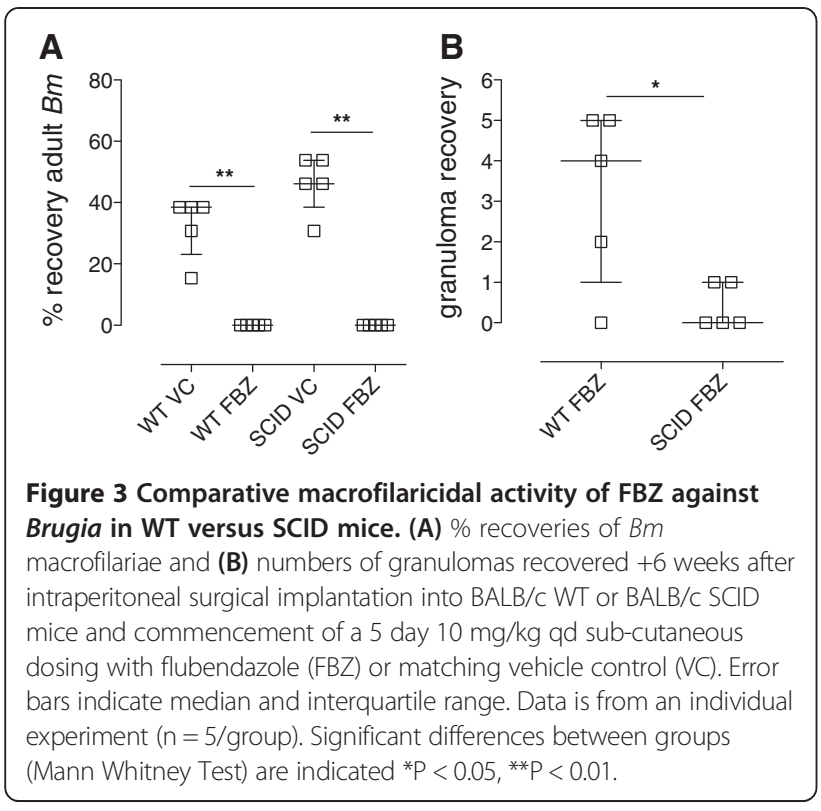

embryogenesis within implanted female Onchocerca uteri was apparent by histological examination, including the presence of inter-uterine stretched mf. Further, released motile microfilariae were evident in the culture medium following ex vivo culture of implanted sub cutaneous onchocercomata derived from 2/2 SCID CB.17 recipients.

\section{Evaluation of the SCID model of onchocerciasis as a pre-clinical macrofilaricidal drug screen}

Because of initial, reproducible, high recoveries of viable male Onchocerca macrofilariae in BALB/c SCID mice, we evaluated this model as an anti-Onchocerca in vivo macrofilaricidal drug screen where survival over a more protracted time frame (4-5 weeks) would be necessary to evaluate macrofilaricide efficacy. We applied the optimised FBZ screen verified in BALB/c SCID Bm implants (Figure 3) to compare suitability of this system for macrofilaricide testing. An increased number of 15 motile male Onchocerca macrofilariae were implanted into each SCID mouse recipient to mitigate against decline in survival over 5 weeks. Also, daily isolates of macrofilariae (from individual cattle hides) were divided equally between recipients consigned to $\mathrm{FBZ}$ or $\mathrm{VC}$ treatments to avoid inter-group bias in quality and age of male worms that might affect survival. Further, to mitigate against inter-group variation in survival rates over a protracted period post-implantation masking macrofilaricidal effects, group sizes were increased to 7-8 mice. Treatment groups were maintained for up to 31 days (4-5 wks post implant) before recovery of parasites (Figure 8). FBZ induced a significant, almost total macrofilaricidal response, with mean survival of $1.67 \%(+/-1.09, \mathrm{n}=8)$, compared with a mean survival of $43.81 \%(+/-11.44, \mathrm{n}=7)$ recovery in the $\mathrm{VC}$ group $(P=0.0089)$. In the treated group, single motile worms were recovered in $2 / 8$ recipients, whilst the other six mice had either an absence of infection or recovery of completely immotile worms. The two motile worms derived from FBZ treated mice were moribund with irregular and retarded 'twitching' motility, compared to the motility rate observed of macrofilariae recovered from $\mathrm{VC}$ mice or of freshly isolated onchocercomata.

We also tested a lead anti-Wolbachia rifamycin antibiotic, rifapentine (RIFAP), for activity against Onchocerca male worms in CB.17 SCID mice. Groups of two mice were given oral doses of RIFAP $(15 \mathrm{mg} / \mathrm{kg})$ or standard suspension vehicle (VC) daily for 14 days, starting from 1-2 days recovery after surgery. Implanted Onchocerca were retrieved +2 weeks after last drug exposure. Total recoveries of Onchocerca macrofilariae were not affected by
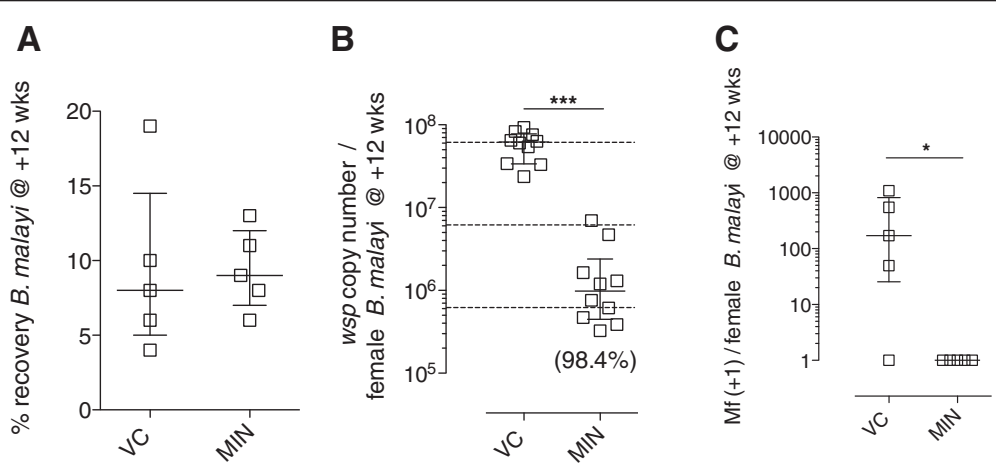

Figure 4 Anti-Wolbachia activity against Brugia macrofilariae in SCID mice. (A) \% recoveries of Bm macrofilariae (B) Wolbachia loads per female $B m$ and (C) numbers of released $m f$ per female $B m$ in BALB/C SCID mice +12 weeks after intraperitoneal infection with $100 \mathrm{BmL} 3$ and +6 weeks after commencement of 4 week oral $25 \mathrm{mg} / \mathrm{kg}$ bid minocycline (MIN) or vehicle control (VC). Error bars indicate median and interquartile range. Dashed horizontal lines in (B) indicate 1 and 2 log reductions compared with median VC levels. Median \% reduction in (B) is indicated in parentheses. Data is from an individual experiment ( $n=5 /$ group; individual worms pooled per group). Significant differences between groups (Mann Whitney Test) are indicated ${ }^{*} \mathrm{P}<0.05,{ }^{* * *} \mathrm{P}<0.001$ 


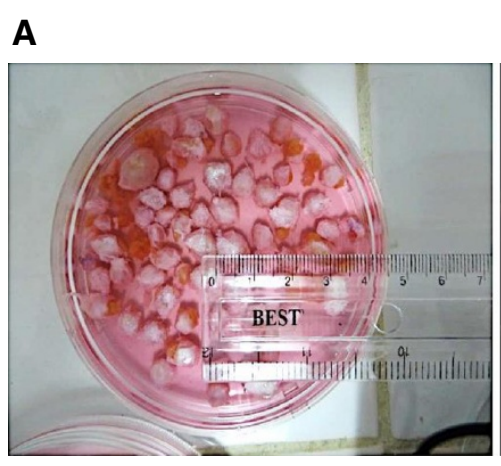

B

C

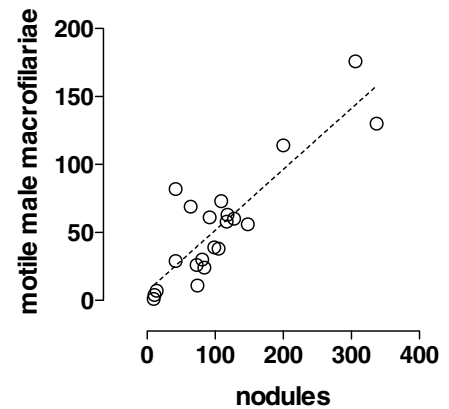

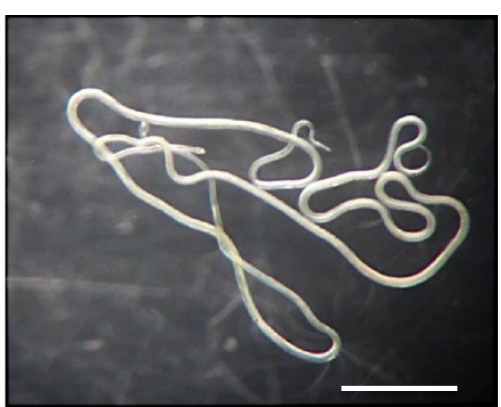

D

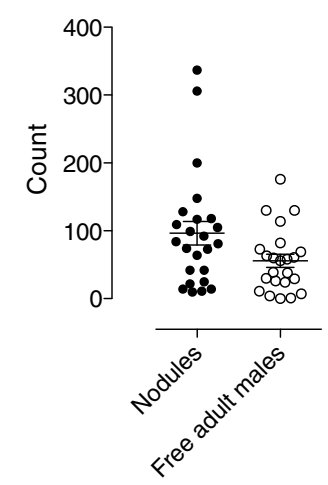

Figure 5 Yields of female and male Onchocerca macrofilariae isolated from naturally infected cattle. A) Typical yield of excised $O$. ochengi onchocercomata from a parasitized cattle hide B) A pair of liberated, motile male 0 . ochengi worms $+4 \mathrm{~h}$ after disruption of onchocercoma (nodule) capsule and culture at $37^{\circ} \mathrm{C} / 5 \% \mathrm{CO}_{2}$. Scale bar $=1 \mathrm{~cm}$. C) Relationship between numbers of onchocercomata recovered per cattle hide and number of motile male $\mathrm{O}$. ochengi harvested. Dashed line is linear regression best fit. D) Recoveries of onchocercomata (closed plots) and male O. ochengi (open plots) per cattle hide $(n=29)$. Error bars are mean $+/-$ SEM.

treatment regimen $(56.65 \%, \mathrm{VC}$ and $58.35 \%$, median recovery, RIFAP; Figure 9A). Numbers of the single copy Wolbachia surface protein (wsp) gene per isolated male Onchocerca marofilariae were quantified as a surrogate measurement of endosymbiont density within nematode tissues (Figure 9B). A highly significant 99\% reduction in Wolbachia number was recorded in Onchocerca macrofilariae obtained from CB.17 SCID mice treated with rifapentine for 14 days $\left(4.6 \times 10^{6}\right.$ median wsp copies, VC $v s$. $0.0452 \times 10^{6}$, RIFAP, $\left.P<0.001\right)$. Wolbachia reductions were preserved when adjusted for potential variation in size and age of adult male Onchocerca derived from cattle onchocercomata, by normalisation to a single copy filarial gene, gst (Figure 9C).

\section{Discussion}

The development of macrofilaricides against onchocerciasis is currently hampered by a lack of a facile preclinical infection model. Extrapolating efficacy of drug candidates against lymphatic filariae in susceptible rodents may not necessarily translate into effective onchocerciasis indications and traditional pre-clinical testing in cattle onchocerciasis does not possess the required throughput to address current demand. For these reasons we decided to develop and validate a small animal model of onchocerciasis. We chose to trial SCID mice as a compatible host for the cattle Onchocerca, O. ochengi. We selected this particular parasite after identifying an abundant and relatively convenient sampling source of O. ochengi macrofilariae in cattle herds derived from the Adamawa region of Northern Cameroon used for commercial meat production. Our experience over a threemonth evaluation period indicated that the prevalence of infected female cattle being moved for slaughter in the South West Province was typically between 5-10\% and that, with around 10-20 cattle being processed daily at a local abattoir, there was frequent availability of infected cow tissues. Because we typically collected >100 O. ochengi macrofilariae from a single hide, this provided an adequate daily supply line of Onchocerca for in vivo drug testing. The implantation of macrofilariae into groups of rodents from a single cow effectively increased capacity 4-5 fold in terms of biological units available for drug testing. Further, considering the much reduced space and costs demanded for long-term husbandry of rodents $v s$ cattle, the rodent model should further facilitate increased 


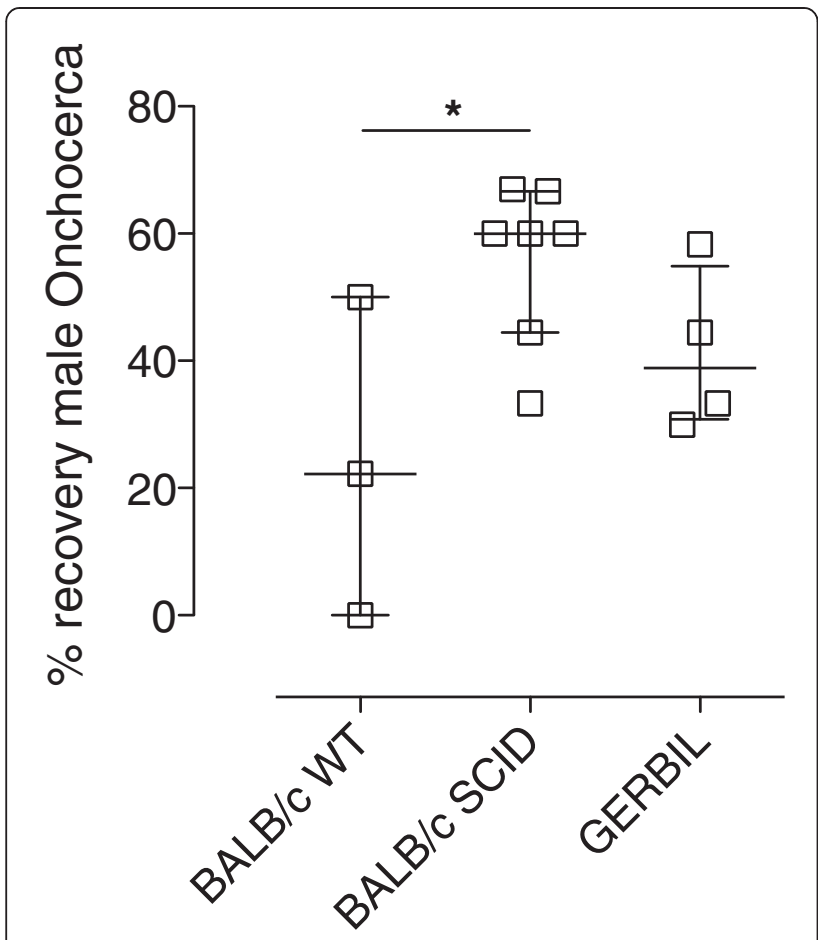

Figure 6 Survival of male Onchocerca macrofilariae in laboratory rodents. Percentage recoveries +15 days post-intraperitoneal implantation with 8-12 isolated, motile male Onchocerca macrofilariae in BALB/C WT, BALB/C SCID and Meriones unguiculatus gerbils. Error bars are median recoveries with interquartile range. For $\mathrm{BALB} / \mathrm{C} S \mathrm{SID}$, data is pooled from two individual experiments $(n=3 / 4)$. For BALB/C WT and Meriones, data is from a single experiment $(n=3 / 4)$. Significant differences between groups (Kruskal-Wallis Test with Dunn's multiple comparison) is indicated ${ }^{*} P<0.05$.

capacity for simultaneous or overlapping Onchocerca drug screening within the same laboratory, in a cost-effective manner. The ability to culture the male $O$. ochengi macrofilariae ex vivo for durations of at least five days, also means that this method of collection could be applied to in vitro testing of novel macrofilaricides, similar to the published male O. gutterosa drug screen [29].
The selection of SCID mice was based on extensive use of this lymphopenic mouse system for xenograft transfers in cancer and malaria chemotherapy pre-clinical testing $[30,31]$ and our own and other laboratory's observations that SCID mice are permissive hosts for non-murine filariae, including full development of the human lymphatic filariae, B. malayi [21] and stage-specific Onchocerca spp. infections [20,22].

A potential caveat to the application of SCID mice for anti-filarial drug screening is the absence of adaptive immune responses, which might be important in interacting with candidate filaricidal compounds to induce macrofilaricidal effects. Whilst it is inconclusive whether inflammatory reactions in patients post-treatment with anti-filarial drugs (e.g. "Mazzoti reactions") are a necessary component in the death of filariae or merely a response to dead and damaged worms [16] and the concomitant liberation of somatic antigens and Wolbachia endobacteria [32,33], the former has been proposed due to a general lack of efficacy of SAFD at physiological levels in vitro against filariae $[34,35]$. Further, various 'immuno-pharmacological' modes of action have been proposed for SAFD, including the prevention of immuno-modulatory secretions from $\mathrm{mf}$ by IVM [36] and the induction of host inducible nitric oxide and cyclooxygenase pathways by DEC [24]. Thus, it was important to validate anti-filarial responses in the SCID model against an immunologically intact WT control, where possible. For this we took advantage of WT BALB/ c mice as an immune competent background strain that, whilst resistant to chronic patent infections, accommodates life cycle stages of $B$. malayi for periods sufficient to evaluate filaricidal drug effects [24,27]. The results of our studies, comparing larvicidal responses of the SAFD: ABZ, DEC and IVM, illustrated no difference in the level of observed efficacy of these reference anti-filarials against infectious Bm L3 larvae, or in the case of IVM, bloodborne $\mathrm{mf}$, in the absence of adaptive immune responses. In fact, for $\mathrm{mf}$, we observed a 2.5 fold higher peripheral circulating $\mathrm{mf}$ and 6.4 fold increased cardiac blood levels in SCID vs

Table 1 Parasitological observations of Onchocerca ochengi onchocercomata post-implantation into SCID mice

\begin{tabular}{|c|c|c|c|c|c|}
\hline Mouse strain/ID & Number/location & Vascularisation & Motile females* & Motile males* & Motile $\mathrm{mf}^{*}$ \\
\hline BALB/C SCID 1 & 4 ip & $4 / 4$ & $4 / 4$ & $2 / 4$ & nd \\
\hline $\mathrm{BALB} / \mathrm{C}$ SCID 2 & 4 ip & $2 / 4$ & $4 / 4$ & $0 / 4$ & nd \\
\hline BALB/C SCID 3 & 4 ip & $0 / 4$ & $4 / 4$ & $0 / 4$ & nd \\
\hline $\mathrm{BALB} / \mathrm{C} \mathrm{SCID} 4$ & 4 ip & $0 / 4$ & $4 / 4$ & $2 / 4$ & nd \\
\hline BALB/C SCID 5 & 4 ip & $1 / 4$ & $0 / 4$ & $2 / 4$ & nd \\
\hline BALB/C SCID 6 & 4 ip & $2 / 4$ & $4 / 4$ & $2 / 4$ & nd \\
\hline CB.17 SCID 1 & $4 \mathrm{sc}$ & $4 / 4$ & $2 / 2$ & $0 / 2$ & yes \\
\hline CB.17 SCID 2 & $4 \mathrm{sc}$ & $4 / 4$ & $3 / 3$ & $1 / 3$ & yes \\
\hline
\end{tabular}

Intraperitoneal implantation (ip), sub cutaneous implantation (sc).

${ }^{*}$ motility assessed by dissection microscope $+4 \mathrm{~h}$ after excision of nodule grafts, disruption of nodule capsule and culture in complete medium at $37^{\circ} \mathrm{C} / 5 \% \mathrm{CO}_{2}$. nd - not done. 


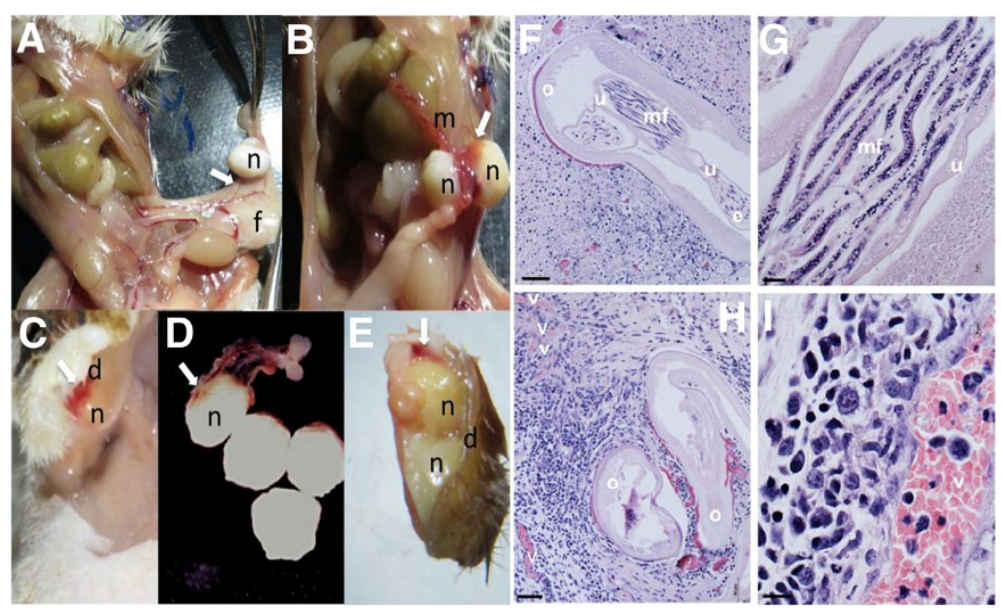

Figure 7 Engraftment of Onchocerca onchocercomata in SCID mice. Engrafted peritoneal (A\&B) or sub cutaneous (C\&D) onchocercomata in situ or excised nodules from the peritoneum (D) or sub cutaneous tissue (E) +7 days after implantation into BALB/C or CB.17 SCID mice. Haematoxylin and eosin staining of sub cutaneous engrafted onchocercomata illustrating embryogenesis (F) and putative murine host vessel-like structures (H). High magnification images of inter-uterine stretched microfilarae (G) and vessel-like structure (I). Key: dermis (d), embryos (e), visceral fat $(\mathrm{f})$, mesentery $(\mathrm{m})$, microfilariae $(\mathrm{mf})$, nodule $(\mathrm{n})$, female Onchocerca ochengi $(\mathrm{o})$, uteris $(\mathrm{u})$, vessel $(\mathrm{v})$. Arrows indicate zones of neovascularisation. Scale bars are $50 \mu \mathrm{m}(\mathrm{F} \& H)$ and $10 \mu \mathrm{m}(\mathrm{G} \& \mathrm{l})$.

WT control groups at 9 days post-tail vein perfusion, which allowed us to discern a more obvious treatment effect of single dose IVM. We also observed similar mf levels in CB.17 SCID mice (data not shown). The elevated persistence of circulating $\mathrm{mf}$ in SCID mice may be due to a lack of initiation of an effective stage-specific adaptive immune response. Certainly, microfilariaemic brugian filariasis patients have hyporesponsive filarial antigenspecific peripheral blood mononuclear cell responses vs amicrofilaraemic infected individuals [37]. Splenic clearance has been demonstrable in controlling bloodborne microfilaraemias in mice [38] and baboons [39] which suggests that SCID mice may have a defective mechanism at this secondary lymphoid organ.

This data extends experiments undertaken in athymic nude mice that demonstrates a lack of a role for $\mathrm{T}$ lymphocytes/lymphocyte 'help' in a DEC mode of action [40] and also suggests $\mathrm{T}$ and $\mathrm{B}$ lymphocyte-mediated adaptive immunity is dispensable for the effects of the benzimidazole (BZ) and macrocyclic lactone families of anti-filarial drugs. Further, a complete FBZ macrofilaricidal response was apparent in both WT and SCID animals using a dose regimen previously reported as effective in the Meriones gerbil Brugia macrofilariae implant model [41]. FBZ macrofilaricidal efficacy generally proceeded without overt leukocytic granuloma formation in SCID mice suggesting that these reactions are a response to dead worms but are not integral to the mode of action of BZ anthelmintics. Whether this extends to other classes of SAFD that have macrofilaricidal activity against $B m$, e.g. DEC and MOX, remain to be evaluated.
We extended our evaluation of the SCID mouse, taking advantage of the full development of Brugia in this immunocompromised system, to assess the appropriateness of its use as a facile anti-Wolbachia drug screen. For this we chose a point of oral administration to drug with the anti-Wolbachia reference tetracycline, MIN, once parasites had undergone the final moult to become immature macrofilariae. At this point in the $B m$ life cycle, Wolbachia numbers have completed a log phase expansion and are representative of levels in mature Brugia (1-5 × 107 Wolbachia per female worm) [25]. Further, initiating anti-Wolbachia drugging at the immature macrofilarial stage allowed us to simultaneously and rapidly discern downstream effects on embryogenesis via initial $\mathrm{mf}$ release, at the immediate onset of patency $(+10-11$ weeks in mice). We could consistently recover $\mathrm{Bm}$ macrofilariae in infected and dosed SCID animals, supporting prior observations of full permissiveness of this model [21] and allowing for reliable production of the required numbers of adult worms and $\mathrm{mf}$ release for assessment of Wolbachia efficacy. Oral MIN administration in infected SCID mice for 28 days reduced Wolbachia loads within female $\mathrm{Bm}$ below the $90 \%$ threshold deemed to irreversibly sterilise filarial tissues in clinical studies, leading to macrofilaricidal effects $[33,42,43]$. The $>90 \%$ levels of depletion observed in female macrofilariae derived from drugged SCID $\mathrm{Bm}$ infections were consistent with in vivo effects reported following protracted tetracycline treatments in patent $\mathrm{Bm}$ infections of gerbils [44] and were reflected in a complete blockade in $\mathrm{mf}$ release in drugged animals. 


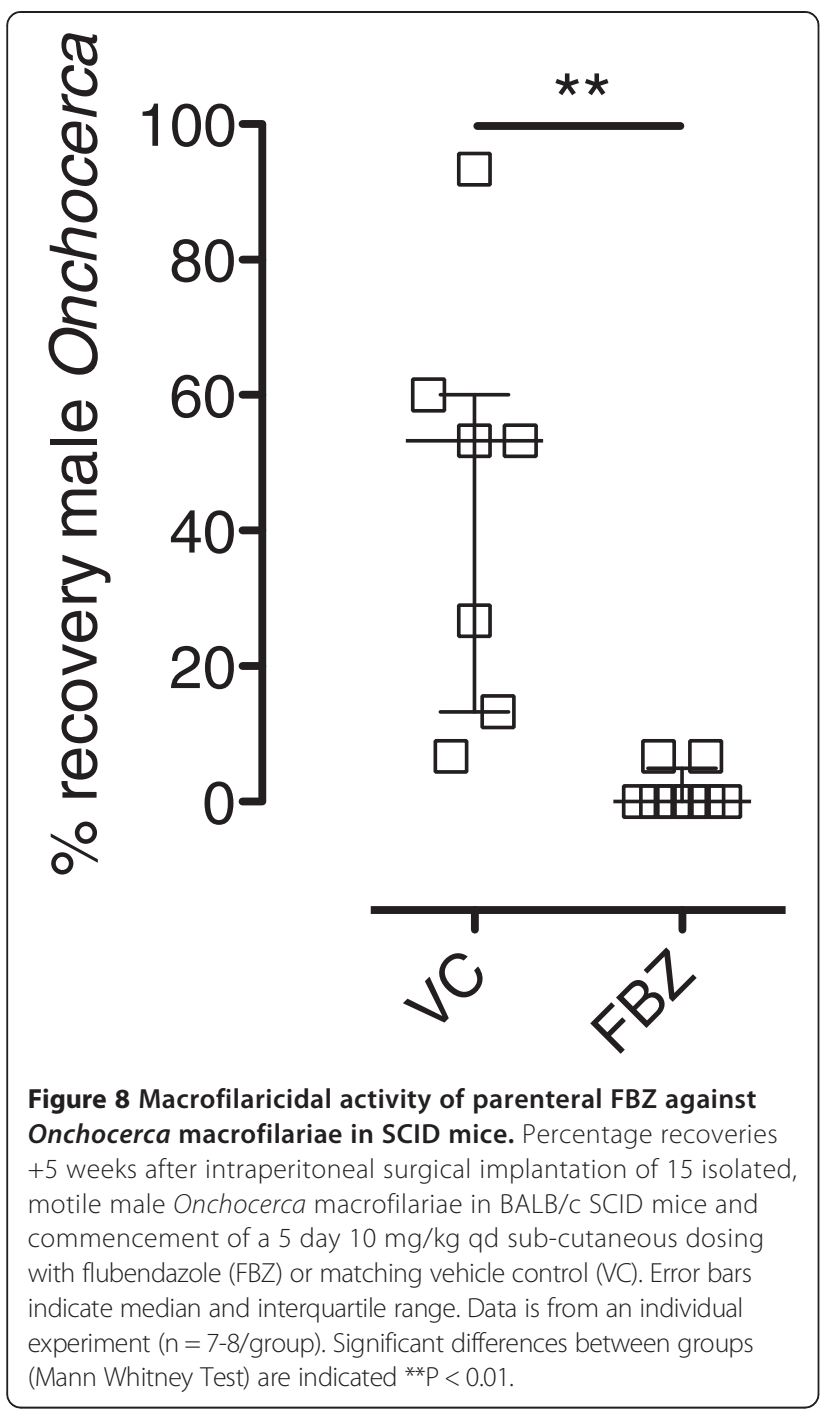

Having validated the SCID model system as suitably responsive to a range of filaricidal reference compounds, we tested survival rates of male Onchocerca macrofilariae in either SCID BALB/c or WT BALB/c mice. The results of a two-week implant pilot study indicated that male Onchocerca survived significantly better in the immunocompromised system, suggesting that adaptive immune responses begin to exert attritional effect on survival by this stage. This is consistent with the kinetics of immuneattrition against larval $\mathrm{Bm}$ infections where adaptive immune responses begin to exert significant filaricidal activity at +2 weeks post-infection [26]. We were also able to preliminarily evaluate survival in the Brugia susceptible outbred rodent, Meriones, which indicated that male Onchocerca macrofilariae could persist at a similar level as within SCID mice. As our focus was on development of a flexible 'pan-filarial' SCID pre-clinical model, we did not investigate survival in Meriones gerbils further. However our initial findings suggest Meriones may also be a suitable laboratory host for male Onchocerca implants and further investigation is warranted to explore the length of persistence in this immune-intact outbred rodent. It is debatable whether macrofilaricides with a total reliance on adaptive immunity would be suitable for indications in chronic filariasis patients who typically display hyporesponsive $\mathrm{T}$ cell profiles to filarial antigens $[37,45,46]$. Furthermore, identification of 'hits' within in vitro culture screening assays $[29,47]$ would fail to identify drugs with a reliance on host adaptive immune responses and as such, these candidates would be suitable for in vivo evaluation in SCID mice. However, should drugs emerge with putative $\mathrm{T}$ or B cell adaptive immune-pharmacological mechanisms, the availability of comparative Bm and Onchocerca macrofilaricide screens in WT mice, SCID mice and gerbils would serve

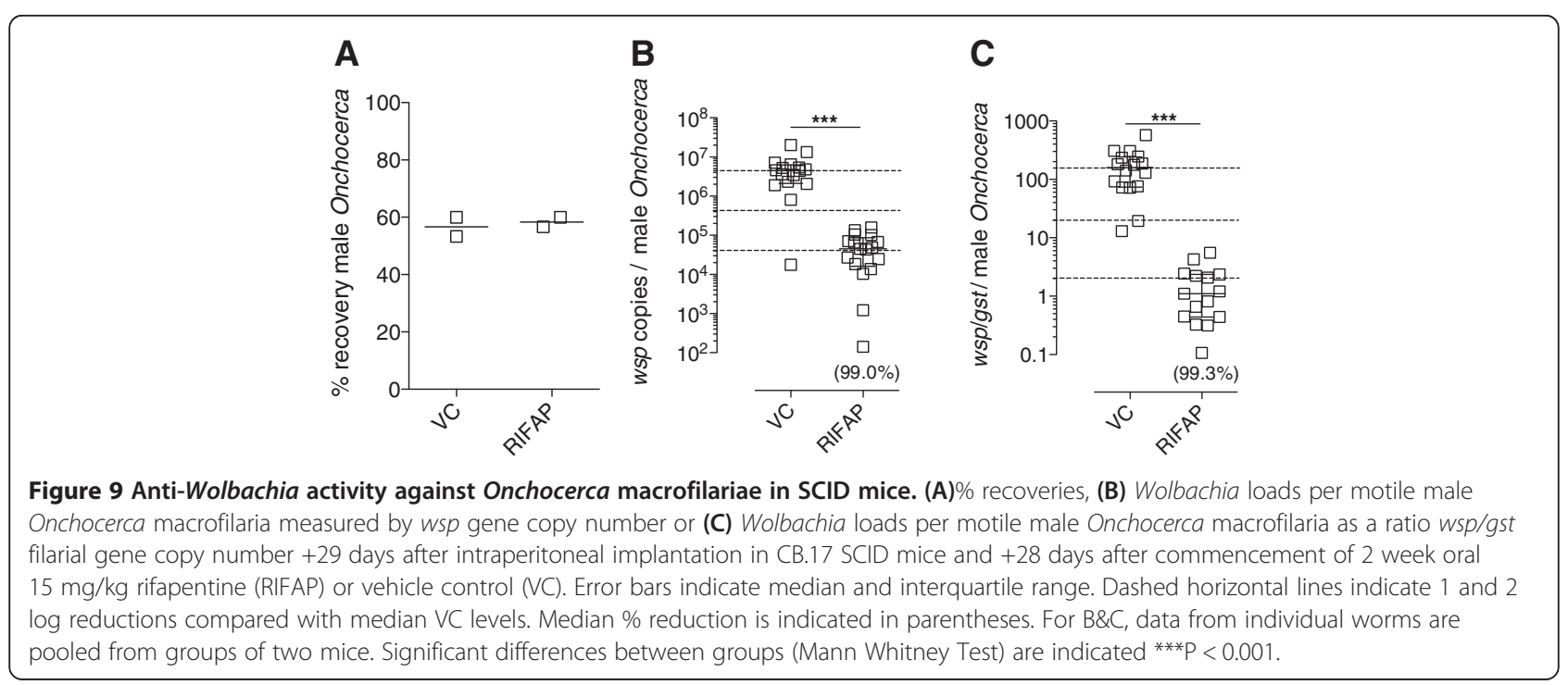


as useful pre-clinical assessment tools to dissect the mode of action and spectrum of anti-filarial effects.

By applying the efficacious FBZ regimen demonstrable to exert macrofilaricidal effects against $B m$ adults, we could reproduce the profound effect of this drug against implanted adult male Onchocerca macrofilariae within SCID mice. This tallies with the reported macrofilaricidal effect of the modified FBZ, UMF078, against $O$. ochengi in the natural cattle host [48]. Thus, our experiment demonstrates 'proof of principle' that the SCID mouse Onchocerca macrofilariae implant model is sufficiently robust to test macrofilaricide activity over a 5 week time frame. Because single, moribund yet motile worms could be isolated in a minority of FBZ treated mice, a slightly extended period may be warranted to determine maximum macrofilaricidal activity. Male macrofilariae were reproducibly recovered from $100 \%$ of 22 SCID mouse recipients in our experiments (discounting FBZ treatments), assessed between 2-5 weeks post implant (mean survival $=49.22 \%+/-5.08$ ). It is probable that more protracted durations of survival are achievable and this requires further assessment.

Confirming our validation experiments with $\mathrm{Bm}$, the male Onchocerca macrofilariae SCID implant model was also assessed as a suitable anti-Wolbachia screen, with strong Wolbachia signal being reliably detected from individual male Onchocerca worms and $>98 \%$ depletions of Wolbachia from filarial tissues recorded following 'goldstandard' oral RIFAP treatment. This is consistent with the rapid effects of the rifamycin class of antibiotics observable in the $B$. malayi infection model in SCID mice (manuscript in preparation).

The matching anatomical site of $\mathrm{Bm}$ adult parasitism and Onchocerca macrofilariae implantation within the peritoneal cavity of the pan-filarial SCID pre-clinical screen, along with availability of both Brugia and Onchocerca macrofilariae ex vivo screening systems, offers a comprehensive suite of pre-clinical tools for robust interrogation of novel macrofilaricide and anti-Wolbachia drugs in development. This is further aided by a high throughput cell screening assay for anti-Wolbachia drug discovery [49]. The pan-filarial murine SCID host described here will therefore provide a useful tool to facilitate the construction of rational PK/PD models and help drive iterative medicinal chemistry to further improve promising classes of drugs. Perhaps the most important advance this model provides is the ability to more accurately discern comparative drug class effects against different genera of medically important filariae in a controlled experimental system. Should the Bm life cycle be established in sub-Saharan African laboratories, this raises the possibility of undertaking dual $\mathrm{Bm}$ and Onchocerca macrofilarial implants within the same host, which would powerfully address the issue of drug effect on different filarial genera.
Given that SCID mice have an impaired mechanism of foreign tissue rejection and are routinely used for grafting human tumours [30] it was hypothesized that $O$. ochengi onchocercomata from the dermis of infected cows could survive upon engraftment into these mice, giving rise to the possibility of developing a murine model containing adult males, females and $\mathrm{mf}$ and keeping the natural anatomical structure of the parasitic niche intact. This would offer a desirable refinement to the male Onchocerca SCID implant model and control for testing drug exposure effects at a more naturalistic site of sub-cutaneous parasitism and/or emulate drug targeting of worms within a complex tissue surrounding of the onchocercoma. For this reason we undertook a pilot study whereby onchocercomata were implanted both into the peritoneal cavity and sub-cutaneously. It was observed that, at both sites, many of the nodules had become attached to host tissues and the majority of nodules had evidence of rapid host neovascularization. Vascularisation with both blood and lymphatic vessels is a consistent feature of $O$. volvulus nodule microarchitecture [50-52]. Whether neovascularisation is actively induced by pro-angiogenic parasite secretions [53] or is part of an innate inflammatory response to foreign material, remains to be resolved. Analogous to solid tumours, that can be successfully targeted by antiangiogenic therapies, neovascularisation is possibly a pre-requisite for more protracted survival, as a source of nutrients and oxygen supply deep within the nodule. Whilst only evaluated for seven days, our pilot data is encouraging; $83 \%$ of the implanted nodules contained motile female macrofilariae and $25 \%$ also yielded motile male macrofilariae ex vivo. Whilst no $\mathrm{mf}$ were observed from the culture of skin from the ear or tail in SCID recipients of sub-cutaneous nodules, motile $\mathrm{mf}$ were liberated after culturing recovered implants. This suggests that female macrofilariae are fertile and producing $\mathrm{mf}$ post-implant in vivo. Because $O$. lienalis $\mathrm{mf}$ show a cumulative increase in recruitment to the skin during the first three weeks following inoculations in SCID mice [22] extended experiments are required to determine the dynamics of $\mathrm{mf}$ recruitment to the skin from implanted nodules. As lymphatic growth into nodular tissue has been proffered as an exit route for $\mathrm{mf}$ migration into the skin [52], this process may be dependent on lymphangiogenic responses developing within implanted nodules.

These experiments are ongoing and require further optimization and refinement to test whether survival can be achieved for longer periods in vivo, and thus whether an onchocercoma xenograft (OX) model could also serve as a platform for the screening of novel therapeutics. Rajan et al. previously described that exposed 'loops' of $O$. volvulus female worms embedded in onchocercomata could remain viable and contain developing $\mathrm{mf}$ for up to $20 \mathrm{wks}$ post-implant in SCID mice [20], demonstrating the longterm feasibility of this approach. We speculate that the 
reduced biomass and less dense extra-cellular matrix of ochengi vs. volvulus onchocercomata may facilitate an increase in perfusion of host solutes, as well as our observations of neovascularization, to support protracted survival of implanted female macrofilariae.

The full development of B. malayi and the protracted survival of implanted $O$. ochengi in SCID mice raises the possibility that this research model might be exploited for drug screening against other medically important filariae. Recently, it has been demonstrated that L. loa can develop to adulthood in immunodeficient mice (BALB/C IL-4R $\left.\alpha^{-/-} / \mathrm{IL}-5^{-/-}\right)$, although patent infections producing circulating $\mathrm{mf}$ were not achieved [54]. Because the availability of a $L$. loa microfilaraemic mouse model would supplement the identification of safe macrofilaricides against onchocerciasis, an ongoing area of investigation is the testing of $L$. loa development and survival within inbred SCID mouse lines.

\section{Conclusions}

SCID mice can be successfully utilised to maintain all life cycle stages of the lymphatic filariae, Brugia malayi and adult stages of the cattle filaria, Onchocerca ochengi with protracted survival. A range of reference antifilarial drugs including macrofilaricides targeting nematode or Wolbachia endosymbionts have been tested against $B m$ and $O$. ochengi in SCID mice. These reference drugs perform with matching efficacy compared with either immune-competent controls or between the two filarial genera. Thus, we have established a 'panfilarial' in vivo pre-clinical tool suitable to screen novel macrofilaricides against both lymphatic and Onchocerca filarial genera of medical importance.

\section{Competing interests}

The authors declare that they have no financial or other competing interests.

\section{Authors' contributions}

$\mathrm{AH}$ designed the study, carried out experimental infections/implantation studies, undertook statistical analyses and assisted in drafting the manuscript. ACG assisted in experimental infections and undertook molecular assays. HT undertook experimental infections, molecular assays and histology analysis. HMM/CNW/KOJA/TDBK assisted in implantation studies. GF/AS/DC assisted in experimental infections. PE assisted in implantation studies. SW/MJT conceived and designed the study. JDT conceived and designed the study, assisted in implant studies, undertook statistical analyses and wrote the manuscript. All authors read and approved the final manuscript.

\section{Acknowledgements}

We thank Professor Charles MacKenzie for helpful advice regarding FBZ dosing and critical appraisal of the manuscript. This work was supported by a Bill and Melinda Gates Foundation (BMGF) funded Grand Challenges Explorations Phase I award (OPP1086755) to JDT, MJT and SW and the BMGF funded AWOL drug development programme (OPP1045261) to LSTM.

\section{Author details}

${ }^{1}$ Department of Parasitology, Liverpool School of Tropical Medicine, Liverpool, UK. ${ }^{2}$ Research Foundation for Tropical Diseases and the Environment, Buea, Cameroon. ${ }^{3}$ Department of Microbiology and Parasitology, Parasite and Vector Research Unit, University of Buea, Buea, Cameroon.
Received: 19 August 2014 Accepted: 2 October 2014

Published online: 24 October 2014

\section{References}

1. Taylor MJ, Hoerauf A, Bockarie M: Lymphatic filariasis and onchocerciasis. Lancet 2010, 376(9747):1175-1185.

2. Babalola OE: Ocular onchocerciasis: current management and future prospects. Clin Ophthalmol 2011, 5:1479-1491.

3. Turner HC, Walker M, Churcher TS, Osei-Atweneboana MY, Biritwum NK, Hopkins A, Prichard RK, Basanez MG: Reaching the London declaration on neglected tropical diseases goals for Onchocerciasis: an economic evaluation of increasing the frequency of ivermectin treatment in Africa. Clin Infect Dis 2014, 59:923-932.

4. Basanez MG, Pion SD, Boakes E, Filipe JA, Churcher TS, Boussinesq M: Effect of single-dose ivermectin on Onchocerca volvulus: a systematic review and meta-analysis. Lancet Infect Dis 2008, 8(5):310-322.

5. Churcher TS, Pion SD, Osei-Atweneboana MY, Prichard RK, Awadzi K, Boussinesq M, Collins RC, Whitworth JA, Basanez MG: Identifying sub-optimal responses to ivermectin in the treatment of River Blindness. Proc Natl Acad Sci U S A 2009, 106(39):16716-16721.

6. Gardon J, Gardon-Wendel N, Demanga N, Kamgno J, Chippaux JP, Boussinesq $M$ : Serious reactions after mass treatment of onchocerciasis with ivermectin in an area endemic for Loa loa infection. Lancet 1997, 350(9070):18-22.

7. Awadzi K: Clinical picture and outcome of serious adverse events in the treatment of Onchocerciasis. Filaria J 2003, 2(Suppl 1):S6.

8. Bird AC, El-Sheikh H, Anderson J, Fuglsang $\mathrm{H}$ : Visual loss during oral diethylcarbamazine treatment for onchocerciasis. Lancet 1979, 2(8132):46.

9. Fobi G, Gardon J, Kamgno J, Aimard-Favennec L, Lafleur C, Gardon-Wendel N, Duke BO, Boussinesq M: A randomized, double-blind, controlled trial of the effects of ivermectin at normal and high doses, given annually or three-monthly, against Onchocerca volvulus: ophthalmological results. Trans Roy Soc Trop Med Hyg 2005, 99(4):279-289.

10. Mackenzie CD, Geary TG: Flubendazole: a candidate macrofilaricide for lymphatic filariasis and onchocerciasis field programs. Expert Rev Anti Infect Ther 2011, 9(5):497-501.

11. Turner JD, Tendongfor $N$, Esum M, Johnston KL, Langley RS, Ford L, Faragher B, Specht S, Mand S, Hoerauf A, Enyong P, Wanji S, Taylor MJ: Macrofilaricidal activity after doxycycline only treatment of Onchocerca volvulus in an area of Loa loa co-endemicity: a randomized controlled trial. PLoS Negl Trop Dis 2010, 4(4):e660.

12. Wanji S, Tendongfor N, Nji T, Esum M, Che JN, Nkwescheu A, Alassa F, Kamnang G, Enyong PA, Taylor MJ, Hoerauf A, Taylor DW: Communitydirected delivery of doxycycline for the treatment of onchocerciasis in areas of co-endemicity with loiasis in Cameroon. Parasit Vectors 2009, 2(1):39.

13. Ash LR: Chronic Brugia pahangi and Brugia malayi infections in Meriones unguiculatus. J Parasitol 1973, 59(3):442-447.

14. Petit G, Diagne M, Marechal P, Owen D, Taylor D, Bain O: Maturation of the filaria Litomosoides sigmodontis in BALB/c mice; comparative susceptibility of nine other inbred strains. Ann Parasitol Hum Comp 1992, 67(5):144-150.

15. Awadzi K, Opoku NO, Attah SK, Lazdins-Helds J, Kuesel AC: A randomized, single-ascending-dose, ivermectin-controlled, double-blind study of moxidectin in Onchocerca volvulus infection. PLoS Negl Trop Dis 2014, 8(6):e2953.

16. Geary TG, Mackenzie CD: Progress and challenges in the discovery of macrofilaricidal drugs. Expert Rev Anti Infect Ther 2011, 9(8):681-695.

17. Taylor MJ, Van Es RP, Shay K, Folkard SG, Townson S, Bianco AE: Protective immunity against Onchocerca volvulus and $O$. lienalis infective larvae in mice. Trop Med Parasitol 1994, 45(1):17-23.

18. Townson S, Dobinson A, Connelly C, Muller R: Chemotherapy of Onchocerca lienalis microfilariae in mice: a model for the evaluation of novel compounds for the treatment of onchocerciasis. J Helminthol 1988, 62(3):181-194.

19. Townson S, Bianco AE, Owen D: Attempts to infect small laboratory animals with the infective larvae of Onchocerca lienalis. J Helminthol 1981, 55(4):247-249.

20. Rajan TV, Nelson FK, Cupp E, Schultz LD, Greiner DL: Survival of Onchocerca volvulus in nodules implanted in immunodeficient rodents. J Parasitol 1992, 78(1):160-163. 
21. Nelson FK, Greiner DL, Shultz LD, Rajan TV: The immunodeficient scid mouse as a model for human lymphatic filariasis. J Exp Med 1991, 173(3):659-663.

22. Folkard SG, Taylor MJ, Butcher GA, Bianco AE: Protective responses against skin-dwelling microfilariae of Onchocerca lienalis in severe combined immunodeficient mice. Infect Immun 1997, 65(7):2846-2851.

23. Griffiths KG, Alworth LC, Harvey SB, Michalski ML: Using an intravenous catheter to carry out abdominal lavage in the gerbil. Lab Animal 2010, 39(5):143-148

24. McGarry HF, Plant LD, Taylor MJ: Diethylcarbamazine activity against Brugia malayi microfilariae is dependent on inducible nitric-oxide synthase and the cyclooxygenase pathway. Filaria J 2005, 4:4.

25. McGarry HF, Egerton GL, Taylor MJ: Population dynamics of Wolbachia bacterial endosymbionts in Brugia malayi. Mol Biochem Parasitol 2004, 135(1):57-67.

26. Rajan TV, Ganley L, Paciorkowski N, Spencer L, Klei TR, Shultz LD: Brugian infections in the peritoneal cavities of laboratory mice: kinetics of infection and cellular responses. Exp Parasitol 2002, 100(4):235-247.

27. Devaney $E$, Howells RE, Smith G: Brugia pahangi in the BALB/C mouse: a model for testing filaricidal compounds. J Helminthol 1985, 59(2):95-99.

28. Lawrence RA: Lymphatic filariasis: what mice can tell us. Parasitol Today 1996, 12(7):267-271.

29. Townson S: The development of a laboratory model for onchocerciasis using Onchocerca gutturosa: in vitro culture, collagenase effects, drug studies and cryopreservation. Trop Med Parasitol 1988, 39(Suppl 4):475-479.

30. Sausville EA, Burger AM: Contributions of human tumor xenografts to anticancer drug development. Cancer Res 2006, 66(7):3351-3354. discussion 3354

31. Vaughan AM, Kappe SH, Ploss A, Mikolajczak SA: Development of humanized mouse models to study human malaria parasite infection Future Microbiol 2012, 7(5):657-665.

32. Keiser PB, Reynolds SM, Awadzi K, Ottesen EA, Taylor MJ, Nutman TB: Bacterial endosymbionts of Onchocerca volvulus in the pathogenesis of posttreatment reactions. J Infect Dis 2002, 185(6):805-811.

33. Turner JD, Mand S, Debrah AY, Muehlfeld J, Pfarr K, McGarry HF, Adjei O, Taylor MJ, Hoerauf A: A randomized, double-blind clinical trial of a 3 -week course of doxycycline plus albendazole and ivermectin for the treatment of Wuchereria bancrofti infection. Clin Infect Dis 2006, 42(8):1081-1089.

34. Bennett JL, Williams JF, Dave V: Pharmacology of ivermectin. Parasitol Today 1988, 4(8):226-228.

35. Maizels RM, Denham DA: Diethylcarbamazine (DEC): immunopharmacological interactions of an anti-filarial drug. Parasitol 1992, 105(Suppl):S49-S60.

36. Moreno Y, Nabhan JF, Solomon J, Mackenzie CD, Geary TG: Ivermectin disrupts the function of the excretory-secretory apparatus in microfilariae of Brugia malayi. Proc Natl Acad Sci U S A 2010, 107(46):20120-20125.

37. Sartono E, Kruize YC, Kurniawan A, Maizels RM, Yazdanbakhsh M: Depression of antigen-specific interleukin-5 and interferon-gamma responses in human lymphatic filariasis as a function of clinical status and age. J Infect Dis 1997, 175(5):1276-1280.

38. Ajendra J, Specht S, Neumann AL, Gondorf F, Schmidt D, Gentil K, Hoffmann WH, Taylor MJ, Hoerauf A, Hubner MP: ST2 deficiency does not impair type 2 immune responses during chronic filarial infection but leads to an increased microfilaremia due to an impaired splenic microfilarial clearance. PLoS One 2014, 9(3):e93072.

39. Orihel TC, Eberhard ML: Loa loa: development and course of patency in experimentally-infected primates. Trop Med Parasitol 1985, 36(4):215-224.

40. Vickery AC, Nayar JK, Tamplin ML: Diethylcarbamazine-mediated clearance of Brugia pahangi microfilariae in immunodeficient nude mice. Am J Trop Med Hyg 1985, 34(3):476-483

41. Surin J, Denham DA: Comparative susceptibility to anthelmintics of Brugia pahangi in jirds infected by different methods. J Helminthol 1990, 64(3):232-238

42. Debrah AY, Mand S, Marfo-Debrekyei Y, Batsa L, Pfarr K, Buttner M, Adjei O, Buttner D, Hoerauf A: Macrofilaricidal effect of 4 weeks of treatment with doxycycline on Wuchereria bancrofti. Trop Med Int Health 2007, 12(12):1433-1441.

43. Hoerauf A, Specht S, Buttner M, Pfarr K, Mand S, Fimmers R, Marfo-Debrekyei Y, Konadu P, Debrah AY, Bandi C, Brattig N, Albers A, Larbi J, Batsa L, Taylor MJ Adjei O, Büttner DW: Wolbachia endobacteria depletion by doxycycline as antifilarial therapy has macrofilaricidal activity in onchocerciasis: a randomized placebo-controlled study. Med Microbiol Immunol 2008, 197(3):295-311.

44. Turner JD, Langley RS, Johnston KL, Egerton G, Wanji S, Taylor MJ: Wolbachia endosymbiotic bacteria of Brugia malayi mediate macrophage tolerance to TLR- and CD40-specific stimuli in a MyD88/ TLR2-dependent manner. J Immuno/ 2006, 177(2):1240-1249.

45. Satoguina J, Mempel M, Larbi J, Badusche M, Loliger C, Adjei O, Gachelin G, Fleischer B, Hoerauf A: Antigen-specific T regulatory-1 cells are associated with immunosuppression in a chronic helminth infection (onchocerciasis). Microbes Infect 2002, 4(13):1291-1300.

46. Akue JP, Devaney E: Transmission intensity affects both antigen-specific and nonspecific T-cell proliferative responses in Loa loa infection. Infect Immun 2002, 70(3):1475-1480

47. Marcellino C, Gut J, Lim KC, Singh R, McKerrow J, Sakanari J: WormAssay: a novel computer application for whole-plate motion-based screening of macroscopic parasites. PLoS Negl Trop Dis 2012, 6(1):e1494.

48. Dec Bronsvoort BM, Makepeace BL, Renz A, Tanya VN, Fleckenstein L, Ekale D, Trees AJ: UMF-078: A modified flubendazole with potent macrofilaricidal activity against Onchocerca ochengi in African cattle. Parasit Vectors 2008, 1(1):18.

49. Johnston KL, Ford L, Taylor MJ: Overcoming the challenges of drug discovery for neglected tropical diseases: the A.WOL experience. J Biomo/ Screen 2014, 19(3):335-343.

50. Smith RJ, Cotter TP, Williams JF, Guderian RH: Vascular perfusion of Onchocerca volvulus nodules. Trop Med Parasitol 1988, 39(Suppl 4):418-421.

51. George GH, Palmieri JR, Connor DH: The onchocercal nodule: interrelationship of adult worms and blood vessels. Am J Trop Med Hyg 1985, 34(6):1144-1148

52. Attout T, Hoerauf A, Denece G, Debrah AY, Marfo-Debrekyei Y, Boussinesq M, Wanji S, Martinez V, Mand S, Adjei O, Bain O, Specht S, Martin C: Lymphatic vascularisation and involvement of Lyve-1+ macrophages in the human onchocerca nodule. PLoS One 2009, 4(12):e8234

53. Higazi TB, Pearlman E, Whikehart DR, Unnasch TR: Angiogenic activity of an Onchocerca volvulus Ancylostoma secreted protein homologue. Mol Biochem Parasitol 2003, 129(1):61-68.

54. Tendongfor N, Wanji S, Ngwa JC, Esum ME, Specht S, Enyong P, Matthaei KI, Hoerauf A: The human parasite Loa loa in cytokine and cytokine receptor gene knock out BALB/c mice: survival, development and localization. Parasit Vectors 2012, 5:43

doi:10.1186/s13071-014-0472-z

Cite this article as: Halliday et al:: A murine macrofilaricide pre-clinical screening model for onchocerciasis and lymphatic filariasis. Parasites \& Vectors 2014 7:472.

\section{Submit your next manuscript to BioMed Central and take full advantage of:}

- Convenient online submission

- Thorough peer review

- No space constraints or color figure charges

- Immediate publication on acceptance

- Inclusion in PubMed, CAS, Scopus and Google Scholar

- Research which is freely available for redistribution 